RBMC

ISSN 2447-9071

doi https://doi.org/10.36414/rbmc.v7i19.111
Contato para correspondência: Leonardo Luiz Borges

E-mail:

leonardo.cbb@pucgoias.edu.br

Conflito de interesse: Não

Financiamento: Recursos próprios

Recebido: 18/11/2021

Aprovado: 10/12/2021

\section{Emprego de ferramentas in silico para avaliação da atividade biológica dos compostos presentes em Curcuma longa}

\section{Use of in silico tools to evaluate the biological activity of compounds present in Curcuma longa}

Matheus Araújo Borges ${ }^{1}$, Cristiano Mendonça Sarkis ${ }^{1}$, Gabriel Elias de Lima Barros ${ }^{1}$, Gabrielly de Souza Correia², Leonardo Luiz Borges ${ }^{1,3}$

\author{
${ }^{1}$ Pontifícia Universidade Católica de Goiás - PUC Goiás \\ ${ }^{2}$ Centro Universitário de Trindade - UNIFIMES \\ ${ }^{3}$ Universidade Estadual de Goiás - UEG
}

\begin{abstract}
Resumo
Esteestudo objetivou apresentaras propriedades químico-biológicas da espécievegetalCurcumalonga L. e detectar as moléculas com possível atividade biológica de interesse. Umlevantamento bibliográfico foi realizado para buscar informações a respeito dos marcadores químicos já identificados para esta espécie. Após identificação dessas estruturas, a codificação das moléculas para realização das predições foi obtida no servidor Pubchem para as análises posteriores. A etapa de triagem de bioatividade para os marcadores químicos foi realizada com os programas SwisSADME, Swiss Prediction e PASS prediction. Os estudos de interação intermolecular para as moléculas bioativas que melhor se encaixam no farmacóforo foram elaborados através de modelos de interação intermolecular com a estrutura macromolecular. Tendo em vista o objetivo do trabalho, a bisdemetoxicurcumina foi a substância em que as atividades preditas apresentaram maiores probabilidades. No repositório Binding Database, foram investigados os 5 ligantes com menores valores de IC50 (5 moléculas mais potentes) capazes de inibir a ação da enzima ácido graxo sintase. Posteriormente, a molécula bisdemetoxicurcumina foi incorporada a este conjunto e, a partir de análises de agrupamento farmacofórico empregando o servidor PharmaGist, as 6 estruturas compartilharam 3 características espaciais. Esse resultado corrobora ahipótese de que abisdemtoxicurcumina exerce atividade biológica sobre a enzimaácido graxo sintase (FASN). As características dos metabólitos ativos da C. Ionga permitem inúmeras possibilidades de interações biológicas, podendo implicar em distintas aplicabilidades clínicas. Estudos futuros são necessários para melhor elucidar essas aplicações.
\end{abstract}

Palavras-Chave: Curcuma; Curcuminoides; Hipolipemiante.

\begin{abstract}
This study aimed to present the chemical-biological properties of the Curcuma longa Linn. and detect molecules prone to have biological activity of interest. A bibliographic survey was carried out to seek information about chemical markers already identified for this species. Afteridentifying these structures, the coding of molecules to perform the predictions was obtained from the Pubchem server for further analysis. The bioactivity screening step forchemical markers was performed with the SwissADME, Swiss Prediction, and PASS prediction programs. Intermolecular Interaction Studies for the bioactive molecules that best fit the pharmacophore were elaborated through models of intermolecular interaction with the macromolecular structure. Bisdemethoxycurcumin was the substance in which the predicted activities showed the highest probabilities. In the Binding Database repository, the 5 ligands with the lowest IC50 values (5 most potent molecules) capable of inhibiting the action of the fatty acid synthase enzyme were investigated. Subsequently, a bisdemethoxycurcumin molecule was incorporated into this set and, from pharmacophoric cluster analysis using the PharmaGist server, as 6 structures shared 3 spatial characteristics.This result supports the hypothesis that a bisdemtoxiccurcumin exerts biological
\end{abstract}


activityon a fatty acid synthase (FASN) enzyme. The characteristics of the active metabolites of C. longa allow countless possibilities of biological interactions, which may imply different clinical applicability. Future studies are needed to elucidate these applications better.

Keywords: Curcuma; Curcuminoids; Hypolipidemic.

\section{Introdução}

Curcuma longa Linn (família Zingiberaceae), conhecida como "açafrão", é um arbusto perene endêmico da Índia, havendo registros de seu uso desde o século I antes da Era Comum (AEC), embora haja referências à planta em manuscritos gregos do século IV AEC. Esta espécie desenvolveu relação com a expansão da civilização, sendo hoje encontrada apenas em regiões de cultivo humano, sem relato de espécimes crescendo em locais selvagens ${ }^{1}$.

A C. longa apresenta quantidades adequadas de óleos voláteis, como turmerone, atlantone e zingiber, além de proteínas, açúcares e resinas, todos compostos que podem auxiliar em suas propriedades terapêuticas. A curcumina se caracteriza como um polifenol insolúvel em água e que consegue se manter estável no pH ácido do estômago. A parte do vegetal com maior utilização é o rizoma que pode ser consumido fresco ou seco. Para fins de conservação, esse rizoma é desidratado e moído, gerando um pó de coloração dourada denominado turmérico. A utilização do turmérico remete às primeiras descrições de $C$. longa, e, historicamente, tem aplicação na culinária, medicina e religião. A curcumina é o componente majoritário dos rizomas de $C$. longa, sendo responsável por cerca de $2 \%$ do peso seco dos rizomas ${ }^{2}$. Atualmente, a curcumina pode ser obtida comercialmente como uma mistura de três componentes: curcumina (CUR, 77\%); desmetoxicurcumina (DMC, 17\%); e bisdesmetoxicurcumina $(B D M C, \sim 3 \%)^{3}$. Na Índia pode-se encontrar esta mistura de curcuminoides na forma de cápsulas, pomadas, unguentos, cremes e curativos para aplicação tópica, misturada ou não com outros componentes. Contudo, a principal utilização destas substâncias ao redor do mundo é na culinária, como componentes do açafrão-da-índia ${ }^{4}$.

Os compostos presentes no turmérico têm sido utilizados também na medicina ayurvédica há séculos, com diferentes finalidades, sendo as mais notáveis como um composto imunomodulador ${ }^{5}$, hepatoprotetor ${ }^{6}$, antitumoral ${ }^{7}$, antioxidante ${ }^{8}$, anti-inflamatório ${ }^{8,9}$ etc. Entretanto, foi relatado que o principal e mais notável efeito terapêutico desta espécie se deve sobretudo à curcumina, presente em altas concentrações na cúrcuma, garantindo a ela um forte papel anti-inflamatório e, também, antiproliferativo ${ }^{10}$. $\mathrm{O}$ tipo de efeito apresentado pela curcumina depende, em parte, da via de administração, de modo que a via oral é a forma mais comum de ingestão, haja vista a sua presença em variados gêneros alimentícios ${ }^{4}$.

Ademais, a C. longa é um antiaterogênico por seu potente efeito redutor de triglicérides (TG) e do colesterol ${ }^{11}$. A redução dos TG pela curcumina foi demonstrada em indivíduos saudáveis que consumiram uma dose baixa dessa substância ${ }^{12}$. A curcumina mostrou-se eficaz, também, como um potente inibidor da oxidação do LDL, e seu efeito hipolipemiante foi demonstrado em ratos diabéticos ${ }^{13}$. Além disso, foi visualizado que o açafrão aumentou o nível de HDL sérico em estudos com animais ${ }^{14}$.

De modo geral, é possível afirmar que estudos terapêuticos têm revelado o potencial medicinal desta planta. As suas concentrações de componentes antioxidantes, antiinflamatórios, analgésicos, antifúngicos, antibacterianos e antivirais permitem uma abordagem ampla de C. longa em estudos científicos, de modo a correlacionar os seus efeitos terapêuticos com uma gama de condições de saúde onerosas à saúde pública mundial. Atualmente, vários estudos estão em andamento para explorar o impacto farmacológico desta espécie no tratamento da doença de Alzheimer, diabetes, osteoartrite, doença cardiovascular, lesões hepáticas e renais e até mesmo o câncer.

Haja vista as potenciais aplicabilidades clínicas da planta, o presente estudo tem por objetivo apresentar as propriedades químico-biológicas dos marcadores químicos presentes na espécie vegetal $C$. longa e detectar as moléculas com propriedades químicas e estruturais propensas a ter atividade biológica empregando ferramentas in silico.

\section{Métodos}

Os metabólitos ativos presentes na espécie C. longa foram identificados por meio de levantamento bibliográfico para buscar informações a respeito dos marcadores químicos já identificados para esta espécie, lançando mão de bases de dados de artigos científicos, incluindo Pubmed, Scielo, Sciencedirect e Periódicos Capes. Após identificação dessas estruturas, a codificação das moléculas para realização das predições foi obtida no servidor Pubchem para as análises posteriores ${ }^{15}$. A etapa de triagem de bioatividade para os marcadores químicos 
foi realizada com os programas SwissADME ${ }^{16}$, Swiss Prediction $^{17}$ e PASS prediction ${ }^{18-20}$. A busca por moléculas bioativas foi realizada na base de dados Binding $D B^{21}$, utilizada para coletar informações sobre a atividade biológica dos compostos encontrados. As estruturas químicas dos marcadores e de moléculas bioativas identificadas nas etapas anteriores foram modeladas em representações 2D e 3D por meio do programa computacional ACD/ChemSketch Freeware Version (Advanced Chemistry Development, Inc.), gerando os arquivos no formato MDL Molfile para cada molécula.

A respeito da determinação da similaridade farmacofórica, as características químicas das moléculas bioativas foram comparadas com as dos marcadores químicos por meio dos arquivos MDL Molfile gerados. Para tal, utilizou-se a abordagem da modelagem por farmacóforo, através do uso do programa Pharmagist ${ }^{22}$. O termo farmacóforo é definido como o arranjo espacial de características químicas que permite um grupo de moléculas interagir com um alvo biológico através de um modo de ligação específico $22-24$.

Os estudos de interação intermolecular para as moléculas bioativas que melhor se encaixam no farmacóforo foram elaborados através de modelos de interação intermolecular com a estrutura macromolecular. Tais modelos podem ser úteis na proposta de elucidar os mecanismos de interação molecular envolvidos no reconhecimento dos marcadores pelas diversas enzimas e receptores encontrados anteriormente.

\section{Resultados}

Em um primeiro momento, foi realizada uma revisão da literatura sobre a espécie vegetal C. longa e foi visto que existem evidências de atividades antitumoral7, anti-inflamatória8,9 e hipolipemiante ${ }^{11,12}$. Os principais compostos encontrados foram curcumim, bisdemetoxicurcumina, a-turmerona, $\beta$-turmerona, ar-turmerona, monoterpenos a-felandreno e 1,8 - cineol $^{25,26}$.

Por meio do programa SwissADME ${ }^{27}$, realizou-se a predição de propriedades farmacocinéticas e procedeu-se a classificação druglikeness, que é a possibilidade do mesmo apresentar absorção pelo trato gastrointestinal e apresentar propriedades semelhantes aos fármacos conhecidos. As estruturas curcumim, bisdemetoxicurcumina, a-turmerona, $\beta$-turmerona, ar-turmerona, monoterpenos a-felandreno e 1,8 -cineol foram classificadas como druglike.

Posteriormente foram realizadas predições pelo servidor PASS Prediction ${ }^{17}$, obtendo-se as probabilidades das moléculas apresentaram as atividades descritas ( $\mathrm{Pa}>0,7)$ (Tab.1). As principais propriedades preditas foram: efeito antihipercolesterolêmico, inibição da sintase gordurosa-acil-CoA, inibição da expressão de H1F1 A, carmativo, anti-inflamatório, antineoplásico, antieczemático e fibrinolítico. Tendo em vista objetivo do trabalho, a bisdemetoxicurcumina foi a substância em que as atividades preditas apresentaram maiores probabilidades, por isso esta estrutura foi selecionada para análise no Pharma Gist.

Tabela 1. Predições realizadas pelo servidor PASS Prediction ${ }^{17}$, com obteção das probabilidades das moléculas apresentarem as atividades descritas ( $\mathrm{Pa}>0,7)$.

\begin{tabular}{|c|c|c|c|}
\hline Substâncias & $\mathrm{Pa}$ & $\mathbf{P i}$ & Atividade \\
\hline Curcurnina & 0,736 & 0,007 & Anti-hipercolesterolêrnico \\
\hline Bisdernetoxicurcurnina & 0,98 & 0,002 & $\begin{array}{c}\text { Inibidor da expressâo } \\
\text { de HIFIA }\end{array}$ \\
\hline Bisdernetoxicurcurnina & 0,844 & 0,004 & $\begin{array}{l}\text { Inibidor da sintase } \\
\text { gordurosa-acil-coA }\end{array}$ \\
\hline Bisdernetoxicurcurnina & 0,733 & 0,007 & Anti-hipercolesterolêrnico \\
\hline Bisdernetoxicurcurnina & 0,704 & o,ols & Anti-innarnatório \\
\hline Alfa-turnerona & 0,82 & 0,003 & Carminativo \\
\hline Alfa-turnerona & $0,77 \mathrm{~s}$ & 0,014 & $\begin{array}{c}\text { Inibidor da expressâo } \\
\text { de HIFIA }\end{array}$ \\
\hline Alfa-turnerona & 0,747 & 0,01 & Fibrinolítico \\
\hline Beta-turnerona & 0,859 & 0,009 & Antieczernático \\
\hline Beta-turnerona & 0,817 & 0,01 & Antineoplásico \\
\hline Ar-turnerona & $0,8 \mathrm{ss}$ & 0,008 & $\begin{array}{c}\text { Inibidor da expressâo } \\
\text { de HIFIA }\end{array}$ \\
\hline Monotepenos e a-felandren & 0,932 & 0,001 & Carminativo \\
\hline 1,8-cineol & 0,777 & 0,004 & Antineoplásico \\
\hline
\end{tabular}

Legenda: Pa (probabilidade do composto estudado ter atividade biológica descrita, "ser ativo"), Pi (probabilidade do composto estudado não ter atividade biológica descrita, "ser inativo")

No repositório Binding Database, foram investigados os 5 ligantes com menores valores de $\mathrm{IC}_{50}$ (5 moléculas mais potentes) capazes de inibir a ação da enzima ácido graxo sintase. Posteriormente, a molécula bisdemetoxicurcumina foi incorporada a este conjunto e, a partir de análises de agrupamento farmacofórico empregando o servidor PharmaGist, as 6 estruturas compartilharam 3 características espaciais (Fig. 2). Esse resultado corrobora a hipótese de que a bisdemtoxicurcumina exerce atividade biológica sobre a enzima ácido graxo sintase (FASN). 


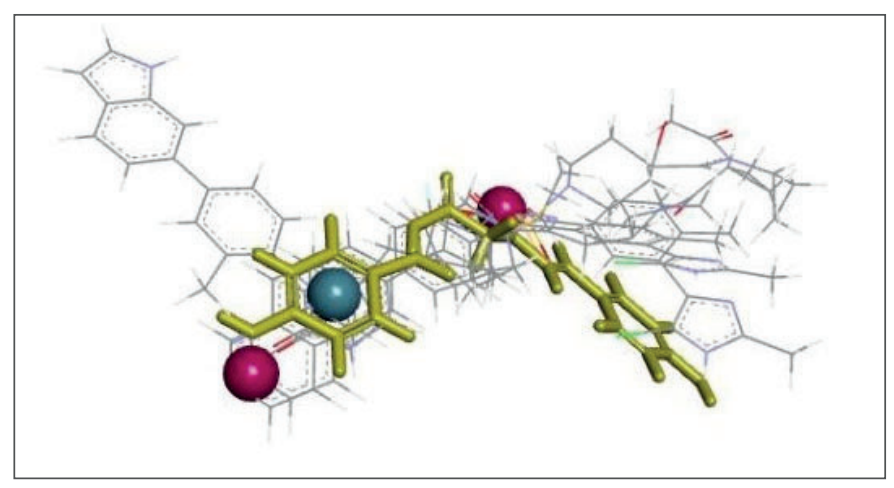

Figura 2. Análise do compartilhamento de grupos farmacofóricos. Bolas em vermelho representam os grupamentos que fazem ligação de hidrogênio. $\mathrm{O}$ anel aromático está representado pela bola azul.

\section{Discussão}

O levantamento de metabólitos ativos da C. longa, com suas respectivas propriedades físico-químicas, classificação druglike, predição de toxicidade, predição da sua atividade anti-hipercolesterolêmico e anti-inflamatória, permitiu a escolha da bisdemetoxicurcumina, a qual apresentou melhores pontuações em todas essas análises, com maior potencial para exercer atividade biológica sobre a enzima ácido graxo sintase.

A bisdemetoxicurcumina, neste estudo, comportou-se como um ligante na estrutura da enzima ácido graxo sintase, permitindo inferir a possível ação anti-hipercolesterolêmica relatada pelo servidor PASS Prediction ${ }^{17}$ e também ser responsável pela ação hipolipemiante da Curcuma sp. L. discutida em estudos prévios $^{11-14}$. Para corroborar essa hipótese, no Binding Database, foram investigadas 5 moléculas mais potentes capazes de inibir a ação da enzima ácido graxo sintase e, ao se comparar as estruturas dessas moléculas à da bisdemetoxicurcumina, 3 características espaciais foram compartilhadas. A enzima ácido graxo sintase é a principal enzima na biossíntese de ácidos graxos. Sua atividade baseia-se na oferta de fosfolipídeos estruturais para as membranas celulares, favorecendo a sua multiplicação. Assim, a inibição dessa enzima, interefere diretamente no metabolismo lipídico, reduzindo a produção desses compostos ${ }^{28}$.

Além dos efeitos anti-hipercolesterolêmico, a bisdemetoxicurcumina apresentou também efeito anti-inflamatório prevista pelo Servidor PASS Prediction e evidenciada em estudo recente ${ }^{17,29}$. A BDMC, por ser um dos componentes da mistura comercial da curcumina ${ }^{3}$, tem contribuição direta na ação contra inflamação dos derivados da C. longa. O mecanismo de ação anti-inflamatório age na cascata do ácido araquidônico, também conhecida como cascata da inflamação, inibindo as moléculas envolvidas no processo inflamatório. Essa inibição envolve mecanismos modulatórios de diversos fenômenos biológicos que interferem nas ativações celulares e nos sinalizadores moleculares, o que tem como efeito final a redução da atividade inflamatória2,30.

Por fim, outro efeito relevante da BDMC é o de inibição do fator indutor de hipóxia 1-alfa (HIF-1a). A hipóxia é uma condição sempre presente no ambiente tumoral devido ao rápido crescimento das células tumorais não suportado por um suprimento sanguíneo adequado. Há evidências crescentes de que a hipóxia desempenha um papel importante na dormência e no metabolismo do câncer, aumentando a atividade da haste e provocando o início e a progressão do câncer. Essa condição pode influenciar a produção do fator indutível por hipóxia (HIF), um fator de transcrição de hélice que está envolvido na carcinogênese e no crescimento do tumor por meio da regulação de genes envolvidos na angiogênese, metabolismo glicolítico e outros mecanismos biológicos, que aumentam de chance de sobrevivência da célula cancerígena mesmo sobre condições de privação de oxigênio ${ }^{31,32}$. Portanto, a bisdemetoxicurcumina, ao inibir a HIF-1 alpha, evitaria a progressão da neoplasia.

\section{Conclusão}

Dentre os principais compostos presentes na C. longa, a BDMC demonstrou ser a molécula com a maior probabilidade de exercer efeitos biológicos sobre a enzima ácido graxo sintase, tendo em vista os parâmetros e predições preconizados para este estudo. Além das ações hipolipemiante e anti-hipercolesterolêmica possibilitadas por essa interação farmacodinâmica, a BDMC também demonstrou potencial anti-inflamatório e propriedades anti-neoplásicas, o que corrobora achados de estudos analíticos e experimentais prévios. As características físico-químicas e estruturais dos metabólitos ativos da C. longa permitem inúmeras possibilidades de interações biológicas, podendo implicar em distintas aplicabilidades clínicas para esses compostos. Testes biológicos futuros com a molécula isolada são necessários, visando encontrar um novo candidato a fármaco hipolipemiante.

\section{Referências}

1. Dalby A. Dangerous tastes: the story of spices. 1 st ed. Oakland: University of California Press; 2001.

2. Sueth-Santiago V, Mendes-Silva GP, Decoté-Ricardo D, Lima MEF de. Curcumin, the golden powder from turmeric: insights into chemical and biological activities. Quim Nova [Internet]. 2015; 38(4):538-552.

3. Goel A, Kunnumakkara AB, Aggarwal BB. Curcumin as "Curecumin": From kitchen to clinic. Biochem Pharmacol. 2008; 75(4):787-809. 
4. Carneiro DM. Ayurveda: Saúde e Longevidade na Tradição Milenar da Índia [Internet]. 1st ed. São Paulo: Pensamento; 2014.

5. Mollazadeh S, Shamsara J, Iman M, Hadizadeh F. Docking and QSAR Studies of 1,4-Dihydropyridine Derivatives as Anti-Cancer Agent. Recent Pat Anticancer Drug Discov. 2017; 12(2):174-185.

6. Momtazi AA, Shahabipour F, Khatibi S, Johnston TP, Pirro M, Sahebkar A. Curcumin as a MicroRNA regulator in cancer: A review. Rev Physiol Biochem Pharmacol. 2016; 171:1-38.

7. Iranshahi M, Sahebkar A, Hosseini ST, Takasaki M, Konoshima T, Tokuda H. Cancer chemopreventive activity of diversin from Ferula diversivittata in vitro and in vivo. Phytomedicine. 2010; 17(3-4):269-273.

8. Sahebkar A. Why it is necessary to translate curcumin into clinical practice for the prevention and treatment of metabolic syndrome? BioFactors. 2013; 39(2):197208.

9. Panahi Y, Sahebkar A, Amiri M, Davoudi SM, Beiraghdar F, Hoseininejad SL, et al. Improvement of sulphur mustard-induced chronic pruritus, quality of life and antioxidant status by curcumin: Results of a randomised, double-blind, placebo-controlled trial. Br J Nutr. 2012; 108(7):1272-9.

10. Sultana S, Munir N, Mahmood Z, Riaz M, Akram M, Rebezov $M$, et al. Molecular targets for the management of cancer using Curcuma longa Linn. phytoconstituents: A Review. Biomedicine and Pharmacotherapy. $2021 ; 135: 111078$.

11. Olszanecki R, Jawień J, Gajda M, Mateuszuk L, Gebska A, Korabiowska $M$, et al. Effect of curcumin on atherosclerosis in apoE/LDLR-double knockout mice. J Physiol Pharmacol [Internet]. 2005 Dec; 56(4):627-35.

12. Disilvestro RA, Joseph E, Zhao S, Bomser J. Diverse effects of a low dose supplement of lipidated curcumin in healthy middle aged people. Nutr J. 2012; 11:79.

13. Ramírez-Tortosa MC, Mesa MD, Aguilera MC, Quiles JL, Baró L, Ramirez-Tortosa CL, et al. Oral administration of a turmeric extract inhibits LDL oxidation and has hypocholesterolemic effects in rabbits with experimental atherosclerosis. Atherosclerosis. 1999; 147(2):371-8.

14. 1Suresh Babu P, Srinivasan K. Hypolipidemic action of curcumin, the active principle of turmeric (Curcuma longa) in streptozotocin induced diabetic rats. Mol Cell Biochem. 1997; 166(1-2):169-75.

15. Kim S, Chen J, Cheng T, Gindulyte A, He J, He S, et al. PubChem 2019 update : improved access to chemical data. 2019; 47(October 2018):1102-9.

16. Daina A, Michielin O, Zoete V. SwissADME: a free web tool to evaluate pharmacokinetics, drug-likeness and medicinal chemistry friendliness of small molecules. Sci Rep [Internet]. 2017 May 3; 7(1):42717.

17. Lagunin A, Stepanchikova A, Filimonov D, Poroikov V. PASS: Prediction of activity spectra for biologically active substances. Bioinformatics. 2000; 16(8):747-8.

18. Poroikov V V, Filimonov DA. How to acquire new biological activities in old compounds by computer prediction. J Comput Aided Mol Des [Internet]. 2002 Nov; 16(11):819-24.

19. Poroikov VV, Filimonov DA, Borodina YV, Lagunin AA, Kos $A$. Robustness of biological activity spectra predicting by computer program PASS for noncongeneric sets of chemical compounds. J Chem Inf Comput Sci [Internet]. 2000; 40(6):1349-55.

20. Sadym A, Lagunin A, Filimonov D, Poroikov V. Prediction of biological activity spectra via the Internet. SAR QSAR Environ Res [Internet]. 2003; 14(5-6):339-47.

21. Liu T, Lin Y, Wen X, Jorissen RN, Gilson MK. BindingDB: A web-accessible database of experimentally determined protein-ligand binding affinities. Nucleic Acids Res. 2007; 35(Database issue):D198-201.

22. Schneidman-Duhovny D, Dror O, Inbar Y, Nussinov R, Wolfson HJ. PharmaGist: a webserver for ligand-based pharmacophore detection. Nucleic Acids Res. 2008; 36(Web Server issue):W223-8.

23. Sheng-Yong Y. Pharmacophore modeling and applications in drug discovery: challenges and recent advances. Drug Discov Today. 2010; 15(11-12):444-50.

24. Wolber G, Seidel T, Bendix F, Langer T. Molecule-pharmacophore superpositioning and pattern matching in computational drug design. Drug Discovery Today. 2008; 13(1-2):23-9.

25. Pal K, Chowdhury S, Kumar S, Chakraborty S, Chakraborty M, Kumar G, et al. Analysis of rhizome colour content, bioactive compound profiling and ex-situ conservation of turmeric genotypes (Curcuma longa L.) from sub- Himalayan terai region of India. Ind Crop Prod [Internet]. 2020; 150(December 2019):112401.

26. Brado G, Dias F, Souza D, Marina R, Andr P, Brugnari T, et al. Curcuma longa L. essential oil composition , antioxidant effect, and effect on Fusarium verticillioides and fumonisin production. Food Control. 2017; 73:806-13.

27. Lipinski CA. Drug-like properties and the causes of poor solubility and poor permeability. J Pharmacol Toxicol Methods. 2000; 44(1):235-49. 
28. Fhu CW, Ali A. Fatty Acid Synthase: An Emerging Target in Cancer. Molecules [Internet]. 2020 Aug 28; 25(17).

29. Jin F, Chen X, Yan H, Xu Z, Yang B, Luo P, et al. Bisdemethoxycurcumin attenuates cisplatin-induced renal injury through anti-apoptosis, anti-oxidant and antiinflammatory. Eur J Pharmacol [Internet]. 2020 May; 874:173026.

30. Marchi JP, Tedesco L, Melo ADC, Frasson AC, França VF, Wietzikoski Sato $S$, et al. Curcuma longa L., o açafrão da terra, e seus benefícios medicinais. Arq Ciências da Saúde da UNIPAR [Internet]. 2016 Mar 30; 20(3).

31. Pezzuto A, Carico E. Role of HIF-1 in Cancer Progression: Novel Insights. A Review. Curr Mol Med [Internet]. 2019 Jan 10; 18(6):343-51.

32. Lequeux A, Noman MZ, Xiao M, Van Moer K, Hasmim $M$, Benoit $A$, et al. Targeting HIF-1 alpha transcriptional activity drives cytotoxic immune effector cells into melanoma and improves combination immunotherapy. Oncogene [Internet]. $2021 \mathrm{Jul}$ 15;40(28):4725-35. 